Basic Science

\title{
e Extracellular Signal-Regulated Kinase 5 in the Cerebrospinal Fluid-Contacting Nucleus Contributes to Neuropathic Pain in Rats
}

Chun-Guang Wang, MD', Si-Yuan Song, MS ${ }^{4}$, Yan-Ling Ding, MS', Shu-Qin Guo, PhD²,

Xin Liu, MS ${ }^{3}$, Shi Hao, BS ${ }^{1}$, Xin Li, BS', Na Chen, BS', Yu Zhang, BS', and Li-Cai Zhang, $\mathrm{PhD}^{4}$

\section{From: ${ }^{1}$ Department of Anesthesiology, The NO.1 Center Hospital Of Bao Ding City, Baoding, 0710oo, Hebei, China; ${ }^{2}$ Department of Endocrinology, The NO.ı Center Hospital Of Bao Ding City, Baoding, 0710oo, Hebei, China; ${ }^{3}$ Department of clinical laboratory, The NO.1 Center Hospital of Bao Ding City, Baoding, 07100o, Hebei, China; ${ }^{4}$ Jiangsu Province Key Laboratory of Anesthesiology, Xuzhou Medical College, Xuzhou, 221002, Jiangsu, China \\ Address Correspondence: Li-Cai Zhang Jiangsu Province Key Laboratory of Anesthesiology Xuzhou Medical College Western HuaiHai Road 84\# Xuzhou Jiangsu, 221002 \\ China \\ Iczhangxzmc@.163.com}

Disclaimer: This work was supported by grants from National Natural Science Foundation of China (NSFC81371243) and Natural Science Foundation of China (BK2012580) to Prof. Zhang.

Manuscript received: 03-10-2015 Accepted for publication: 04-27-2015

Free full manuscript: www.painphysicianjournal.com
Background: The activation of mitogen-activated protein kinases (MAPKs) have been observed in synaptic plasticity processes of learning and memory in neuropathic pain. Cerebrospinal fluidcontacting nucleus (CSF-CN) has been identified with the onset and persistence of neuropathic pain. However, whether extracellular signal-regulated protein kinase 5 (ERK5), a member of MAPKs, in CSF-CN participates in neuropathic pain has not been studied yet.

Objective: The aim of the present study was to identify the role of ERK5 in CSF-CN on the formation and development of neuropathic pain, and to investigate its possible mechanism.

Study Design: Controlled animal study.

Setting: University laboratory.

Methods: After a chronic constriction injury $(\mathrm{CCl})$ model was produced, BIX02188 was dissolved in $1 \%$ DMSO and injected into the lateral ventricles LV in a volume of $3 \mu \mathrm{l}$ with different doses $(0.1 \mu \mathrm{g}, 1 \mu \mathrm{g}, 10 \mu \mathrm{g})$. Mechanical allodynia and thermal hypersensitivity behavioral test, immunofluorescence, and western blot technique were used in this research.

Result: Following $\mathrm{CCl}$, mechanical allodynia and thermal hypersensitivity were developed within a day, peaked at 14 days, and persisted for 21 days. ERK 5 was remarkably activated by $\mathrm{CCI}$ in CSFCN. Moreover, selective inhibiting of p-ERK5 expression in CSF-CN by BIX02188 could significantly relieve $\mathrm{CCl}$-induced mechanical allodynia and thermal hypersensitivity, accompanying with the decreased phosphorylation of CAMP response-element binding protein (CREB) in CSF-CN.

Limitations: More underlying mechanism(s) of the role of ERK5 in CSF-CN on the formation and development of neuropathic pain will be needed to explore in future research.

Conclusion: These findings suggest activation of ERK5 in CSF-CN might contribute to the onset and development of neuropathic pain and its role might be partly accomplished by $p$-CREB.

Key words: Neuropathic pain, extracellular signal-regulated kinase 5, distal cerebrospinal fluidcontacting neurons, cerebrospinal fluid-contacting nucleus, chronic constriction injury, CAMP response-element binding protein

Pain Physician 2015; 18:E1073-E1081 europathic pain, defined as pain caused by an injury of the somatosensory system at the peripheral and central level, is characterized by allodynia, thermal hyperalgesia, and spontaneous pain (1-2). To date, despite considerable progress, the molecular and cellular mechanisms underlying neuropathic pain are not yet completely understood, and, thus, the treatment for it remains a challenge. 
Extracellular signal-regulated protein kinase 5 (ERK5), a member of mitogen-activated protein kinases (MAPKs) family, contains a Thr-Glu-Tyr dual phosphorylation motif of the ERK1/2-type MAP kinase. However, it has a unique loop-12 structure and an unusually large C-terminal non-kinase domain, making ERK5 quite different from ERK1/2 (3). Accumulative evidence indicates that ERK5 is involved in neuronal plasticity, including learning and memory, drug addiction, and pain hypersensitivity (4-8). Activation of ERK5 in rat spinal dorsal horn and dorsal root ganglion (DRG) remarkably increases by nerve injury, and antisense knockdown of ERK5 suppresses nerve injury-induced hyperalgesia (5). Moreover, inflammatory pain induced by injection of complete Freund's adjuvant into rat hind paw produces notable activation of ERK5 in dorsal root ganglion and spinal dorsal horn neurons $(4,8)$. All of these results have indicated that ERK5 might play an important role in the development and maintenance of pathological pain.

The cerebrospinal fluid-contacting neurons are a peculiar type of neurons in the central nervous system. According to the cytologic positions of the neurons, they can be divided into 3 types: an intraependyma neuron which lines the walls of ventricles and the central canal of the spinal cord, a supraependyma cell which is subjacent to the ependymal, and distal cerebrospinal fluid-contacting neurons (dCSF-CNs) whose bodies are in the parenchyma of the brain and whose processes extend into the CSF in the cavity of the ventricle in the central nervous system (9-10). Horseradish peroxidaseconjungated toxin subunit B (CB-HRP), which could be absorbed by dCSF-CNs but not pass through the tight juncture of the ependyma, was used to be a tracer to understand the function of dCSF-CNs (10). Through this approach and morphological methods, we found that dCSF-CNs were a distinct group from other neurons, whose bodies are mainly located in the ventral periaqueductal central gray (PAG) of the brainstem and whose processes penetrate the ependymal cells and stretch into the CSF. In view of the anatomical and morphological characteristics, we named them cerebrospinal fluid-contacting nucleus (CSF-CN) $(6,11)$. For decades, substantial evidence has established that multiple sites are closely related to neuropathic pain, including the somatosensory cortex, dorsal thalamus, rostral anterior cingulate cortex (rACC), amygdale nucleus, prefrontal cortex (PFC), and spinal cord (12-17). However, our recent research revealed that CSF-CN, as a connection between the parenchyma of the brain and the CSF sys- tems, might play an important role in neuropathic pain (18-20). Accordingly, the aim of the present study was to determine whether the activation of ERK5 in CSF-CN contributes to neuropathic pain.

\section{Methods}

\section{Subjects}

All experiment protocols were approved by the Animal Care and Use Committee of Xuzhou Medical College and were in accordance with the ethical guidelines of the International Association for the Study of Pain (21). The animals were provided by Experimental Animal Center of Xuzhou Medical College. Male Sprague-Dawley rats $(220$ - $250 \mathrm{~g})$ were housed on a $12 / 12 \mathrm{~h}$ light/dark cycle, with free access to food and water.

\section{Chronic constriction injury model}

The chronic constriction injury $(\mathrm{CCl})$ model was produced following the method of Benntt and Xie (22). Rats were anesthetized with sodium pentobarbital (50 $\mathrm{mg} / \mathrm{kg}$, intraperitoneal) and the right sciatic nerve was exposed by the blunt preparation of connective tissue at the mid-thigh level, proximal to the sciatic trifurcation. Four sterile 4-0 chromic gut ligatures were loosely tied so as to barely constrict blood flow. In sham-operated animals, the sciatic nerve was exposed but not ligated. The skin was sutured and the anesthesia discontinued. The rats were transferred to a recovery cage until they showed signs of locomotive competence and a reproducible righting reflex.

\section{Measurement of Thermal Hyperalgesia}

The II TC Plantar Analgsia Meter (IITC Life Science Inc., CA) was used to measure paw withdrawal latency, as described by Hargreaves et al (23). Rats were placed in plastic chambers $(7 \times 9 \times 11 \mathrm{~cm})$ to adapt to the environment for one hour before testing. The radiant heat was directed to the plantar surface of each hind paw that was flushed against the glass. The nociceptive endpoints in the radiant heat test were the characteristic lifting or licking of the hind paw. The time to the endpoint was considered the paw-withdrawal latency (PWL). An automatic 25 second cutoff was used to prevent tissue damage. Thermal stimuli were delivered 3 times to each hind paw at 5-minute intervals.

\section{Measurement of Mechanical Allodynia}

Mechanical allodynia was assessed with electronic 
Von Frey filaments (II TC Life Science Inc., CA). Rats were placed in individual plastic boxes $(20 \times 25 \times 15 \mathrm{~cm})$ on a metal mesh floor and allowed to acclimate for one hour before testing. The filaments were presented perpendicularly to the plantar surface, and brisk withdrawal or paw flinching were considered as positive responses, the digital number presented on the monitor was recorded as the paw withdrawal threshold (PWT).

\section{Drug Application}

Rats were anesthetized with sevoflurane and the head fixed in a stereotaxic instrument. Forty-eight hours before they were perfused for immunofluorescence, a 3- $\mu$ l volume of 30\% CB-HRP (Sigma) was injected into one of the rat's lateral ventricles (LV) according to stereotaxic coordinates (Bregma: $-1.2 \pm 0.4 \mathrm{~mm}$, Depth: $3.2 \pm 0.4 \mathrm{~mm}$, Right of median sagittal plane: $1.4 \pm 0.2 \mathrm{~mm}$ ). BIX02188, the latest selective inhibitor of ERK5, was purchased from Boehringer Ingelheim (Ridgefield, CT), which was dissolved in $1 \%$ DMSO and injected into the LV in a volume of $3 \mu \mathrm{l}$ with different doses $(0.1 \mu \mathrm{g}, 1 \mu \mathrm{g}, 10 \mu \mathrm{g})$, the control for BIX02188 administration was $1 \%$ DMSO.

\section{Immunofluorescence}

Forty-eight hours following CB-HRP injection, rats were deeply anesthetized with sevoflurane and perfused with $150 \mathrm{~mL}$ of phosphate buffered saline (PBS) (0.01 M PBS, pH 7.4), followed without interruption by $4 \%$ paraformaldehyde in $0.2 \mathrm{M}$ phosphate buffer (300 $\mathrm{ml}, \mathrm{pH}$ 7.4). The brainstem was removed immediately and post-fixed for $4-6$ hours at $4^{\circ} \mathrm{C}$, then cryoprotected by immersion for $24-48$ hours in sucrose gradients $(5 \%$, $10 \%, 15 \%, 20 \%$, and $30 \%$ ) with $0.01 \mathrm{~mol} / \mathrm{L}$ PBS at $4{ }^{\circ} \mathrm{C}$. The brainstem embedded with OCT at $-20^{\circ} \mathrm{C}$ and sectioned on a cryostat (Leica CM1900, Germany) at $40 \mu \mathrm{m}$ in the transverse plane.

In order to determine the expression of $\mathrm{p}$-ERK5 in CSF-CN, the frozen sections were collected in PBS. Following 3 washes in PBS, sections were incubated in PBS with $0.3 \%$ triton $\mathrm{X}-100$ for $48-72$ hours at $4^{\circ} \mathrm{C}$ with a goat anti-cholera toxin B-subunit (1:200, Sigma) and in rabbit anti-p-ERK5 (1:500, Thermo). After rinsing in PBS, sections were incubated in donkey anti-goat IgG conjugated to Texas Red (1:400, Abcam), and in donkey anti-rabbit IgG conjugated to fluorescein isothiocyanate (FITC) (1:400, Abcam) in the dark for 2 hours at room temperature. Finally, sections were rinsed, mounted, and cover slipped with glycerol containing $2.5 \%$ of antifading agent DABCO (1, 4-di-aza-bi-cyclo-2, 2, 2-octane, Sigma) and stored at $-20^{\circ} \mathrm{C}$ in the dark. Tissue sections were examined using laser scanning confocal microscopy (Leica, Germany) to identify dCSF-CNs labeled with TR and p-ERK5 labeled with FITC.

\section{Western Blot}

Rats were sacrificed and the CSF-CN samples were quickly extracted under deep isoflurane anesthesia and stored in liquid nitrogen. Tissue samples were homogenized in $50 \mathrm{mM}$ RIPA buffer $(20 \mu \mathrm{l} / \mathrm{mg})$, containing protease inhibitor cocktail and phosphatase inhibitor cocktail. Lysates were centrifuged at $14,400 \mathrm{~g}$ for 60 minutes at $4{ }^{\circ} \mathrm{C}$, and the concentration of protein in each sample (supernatant) was determined by the Lowry method. Samples with equal amounts of protein were then separated by $10-20 \%$ PAGE, and the resolved proteins were electro-transferred to nitrocellulose membrane. Membranes were incubated with $5 \%$ nonfat milk with for at least 60 minutes at room temperature and incubated with the following primary antibodies: p-ERK5, ERK5 (1:1000; Cell Signaling Technology) and $p-C R E B, C R E B$ (1:500; SAB) at $4{ }^{\circ} \mathrm{C}$ overnight. The membranes were extensively washed with Tris Buffered Saline Tween (TBST) and incubated for 2 hours with the secondary antibody conjugated with alkaline phosphatase (1:1000) at room temperature. The immune complexes were detected by using a NBT/BCIP assay kit (Sigma). Western blot densitometry analysis of signal intensity was performed using Adobe Photoshop software (Adobe, San Jose, CA), and phosphorylation levels of ERK5 from densitometry were normalized to total ERK5. The blot density from control groups was set as $100 \%$.

\section{Statistical Analyses}

For statistical analysis, GraphPad Prism 5 (GraphPad Software, USA) was used. Data were expressed as mean \pm SEM. Statistical comparison of more than 2 groups was performed using one-way analysis of variance (ANOVA) followed by a Tukey's post hoc test. The significance of any differences in behavior test was assessed using 2-way ANOVA (within-subject factor:time; between-subjects factor:treatment). A value of $P<0.05$ was considered as statistically significant.

\section{Results}

\section{Chronic constriction injury induced nociceptive behavioral and activation of ERK5}

$\mathrm{CCl}$, a well-characterized nerve injury model, was used to discuss neuropathic pain in this study. The pain 
threshold of PWT and PWL decreased after CCI surgery and reached the lowest point at 14 days post-surgery ( $* P$ $<0.05$ at 1 day, ${ }^{* *} P<0.01$ from 3 to 21 days, $\mathrm{CCl}$ group vs sham group; Fig. 1). Thus, 14 days post-operation, as a critical time point, was chosen for drug application and the collection of CSF-CN samples in this research. However, the sham group rats did not exhibit mechanical allodynia and thermal hyperalgesia. In the present study, ERK5 activation was evaluated by detecting the expression of $\mathrm{p}$-ERK5 via immune-fluorescence analysis. The results revealed that the expression of $p$-ERK5 in CSF-CN increased in the $\mathrm{CCl}$ group at 14 days post-CCI compared with the sham operation rats $(* * P<0.01$, compared with sham group; Fig. 2).

Intra-lateral ventricles injection BIX02188 attenuated chronic constrictive injury-induced mechanical allodynia and thermal hypersensitivity.

To clarify the analgesic property of BIX02188, it was intra-lateral ventricles injected to $\mathrm{CCl}$ rats at surgery day 14 . The improvement of mechanical allodynia and thermal hypersensitivity was measured at 30 minutes after intra-lateral ventricles injection of BIX02188, and the most significant reduction was detected at one hour after intra-lateral ventricles injection of BIX02188. Thus, one hour after intra-lateral ventricles injection, as a critical time point, was used for behavior test in this study. The relief was maintained at least up to 4 hours and returned to baseline levels at 8 hours after intralateral ventricles injection of BIX02188 $(* * P<0.01$ at 0.5 h, 1 h, 2 h, $* P<0.05$ at 4 h, vs. 0 h; Fig. $3 A$ ). To further determine the pre-treatment effect of BIX02188 on the improvement of mechanical allodynia and thermal hypersensitivity, consecutive intra-lateral ventricles injection of BIX02188 $(0.1 \mu \mathrm{g}, 1 \mu \mathrm{g}, 10 \mu \mathrm{g})$ before $\mathrm{CCl}$ and on days $1,3,5$, and 7 after $\mathrm{CCl}$ were implemented. One hour after intra-lateral ventricles injection, behavior test was carried out. The result of PWT and PWL indicated that pre-treatment with BIX02188, not DMSO, could attenuate remarkably the mechanical allodynia and thermal hypersensitivity in a dose dependent manner $(* * P<0.01, * P<0.05, \mathrm{BIX} 02188(1 \mu \mathrm{g}, 10 \mu \mathrm{g})+\mathrm{CCl}$ vs DMSO+CCl group; Fig. $3 \mathrm{~B})$.

Intra-lateral ventricles injection of BIX02188 reduced the expression of $\mathrm{p}$-ERK5

Western blot assay revealed that p-ERK5 in CSF-CN increased in $\mathrm{CCl} 14$ day rats compared with sham group. Intra-lateral ventricles consecutive administration of BIX02188 every day, not DMSO, reduced the p-ERK5 expression in CSF-CN of $\mathrm{CCl}$ rats. However, the total ERK5 expression was not altered in any state (Fig. 4).

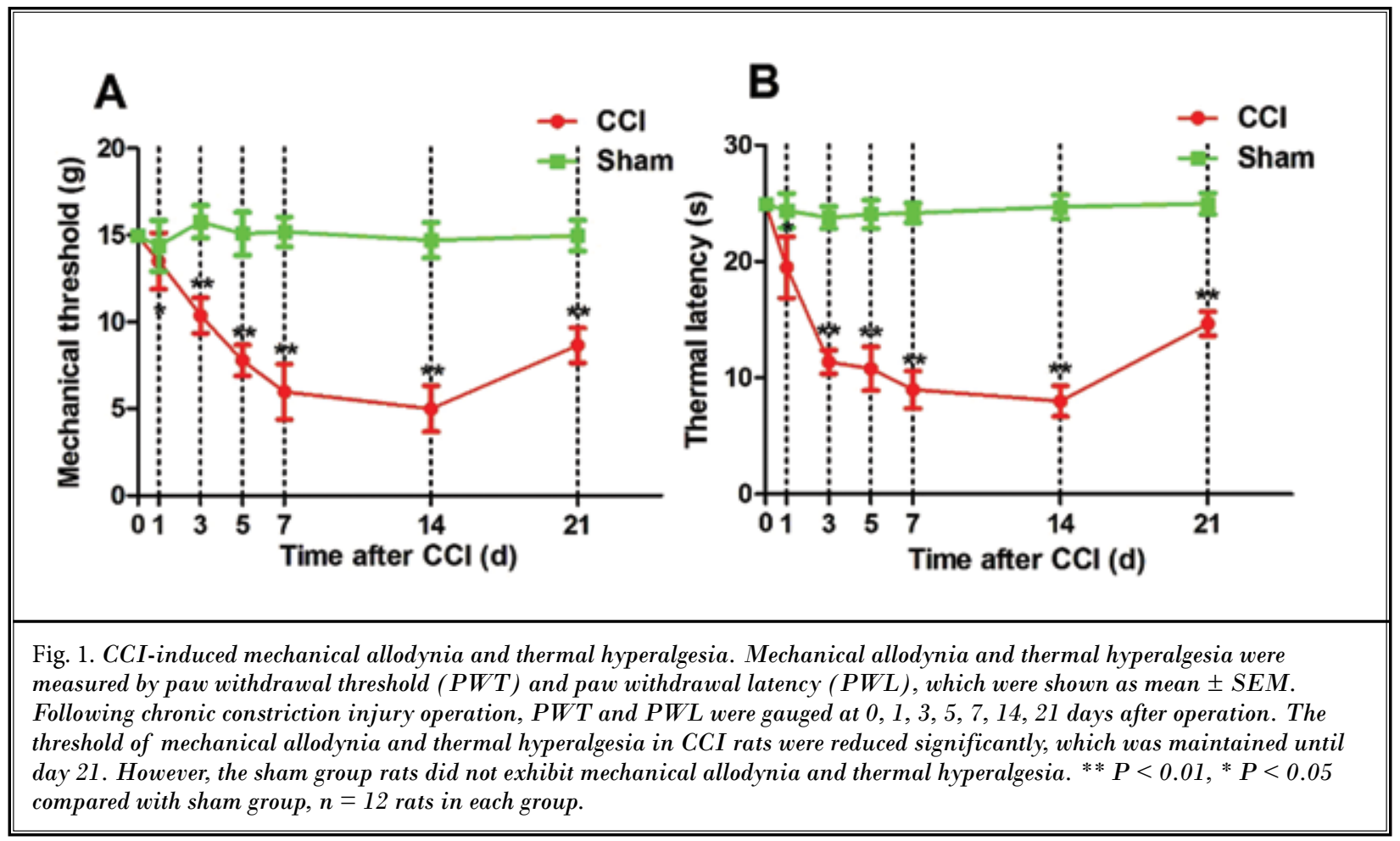



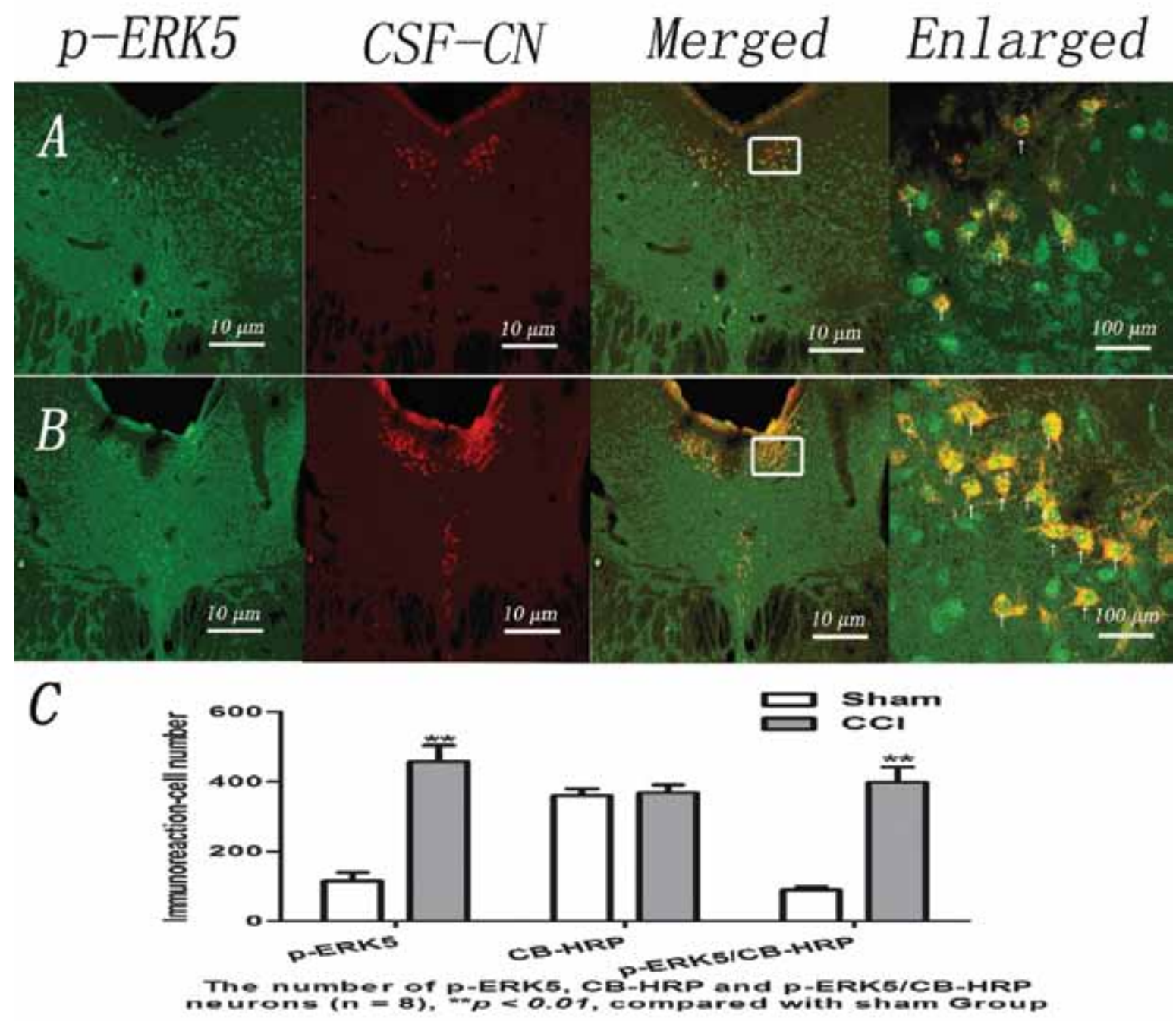

Fig. 2. The expression of p-ERK5 in CSF-CN was increased in the CCI 14 day rats. The dCSF-CNs, which cell bodies were in the parenchyma and the processes were extended into the CSF, were traced by CB-HRP, and labeled in red. P-ERK5 positive neurons were in green and $p$-ERK5/CB-HRP double-labeled neurons were in yellow. Photomicrograph (B) depicts p-ERK5expression in the CCI day 14 group rats, which is significantly higher than the sham group rats $(A)$. Graph $(C)$ depicts the numbers of neurons labeled with $p$-ERK5, CB-HRP and $p$-ERK5/CB-HRP in every 12 sections of 2 groups. ${ }^{* *} P<0.01$ compared with sham group, $n=8$ rats in each group.

Expression of $\mathrm{p}$-CREB was decreased by intra- lateral ventricles injection of BIX02188

Western blot result showed that $\mathrm{p}$-CREB highly expressed in CSF-CN of $\mathrm{CCl} 14$ day rats. Intra-lateral ventricles consecutive administration of BIX02188 every day, not DMSO, could significantly inhibit the increase of $p$-CREB. However, the total CREB expression was not altered in any state (Fig. 5).

\section{Discussion}

The dCSF-CNs are mainly located in the ventral PAG of the brainstem, and the bodies are located in the parenchyma of the brain and processes extend into the CSF in the cavity of the ventricle. According to their anatomical characteristics, the CSF-CN may play a vital function in the substance and signaling transport, modulating between the brain and CSF (10). In patho- 


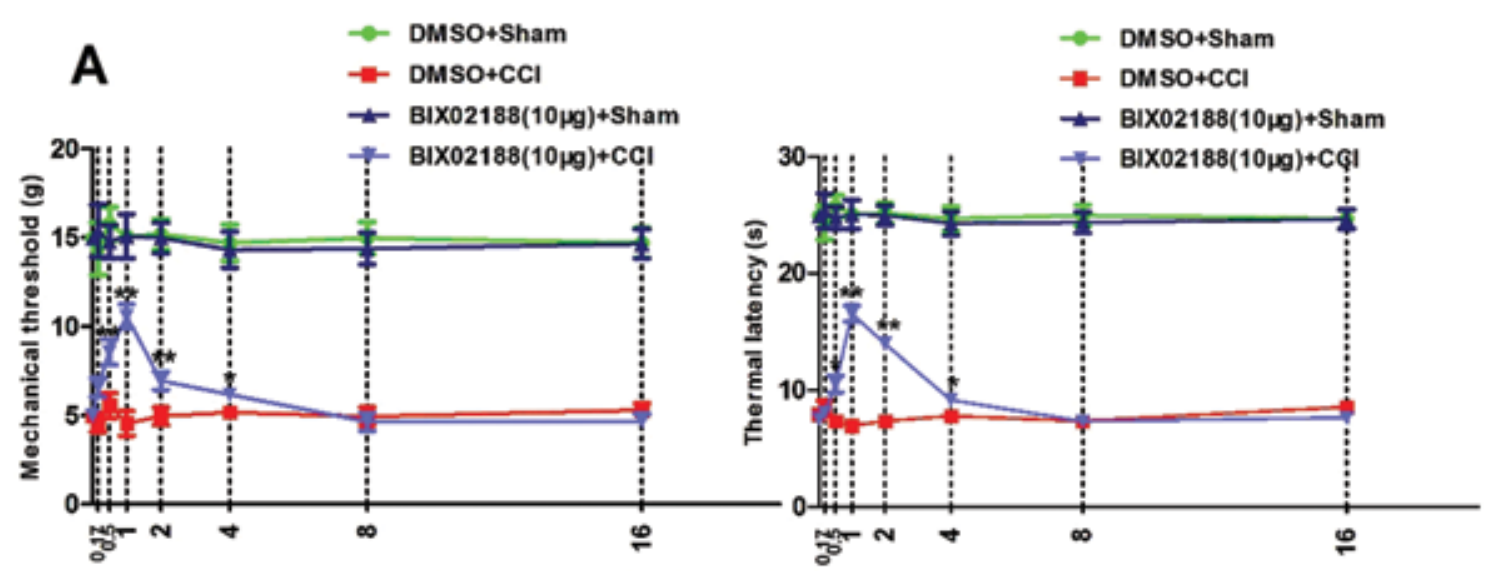

Time after intra-CSF-CN infusion of BIX02188 at day 14 (h) Time after intra-CSF-CN infusion of BIX02188 at day 14 (h)
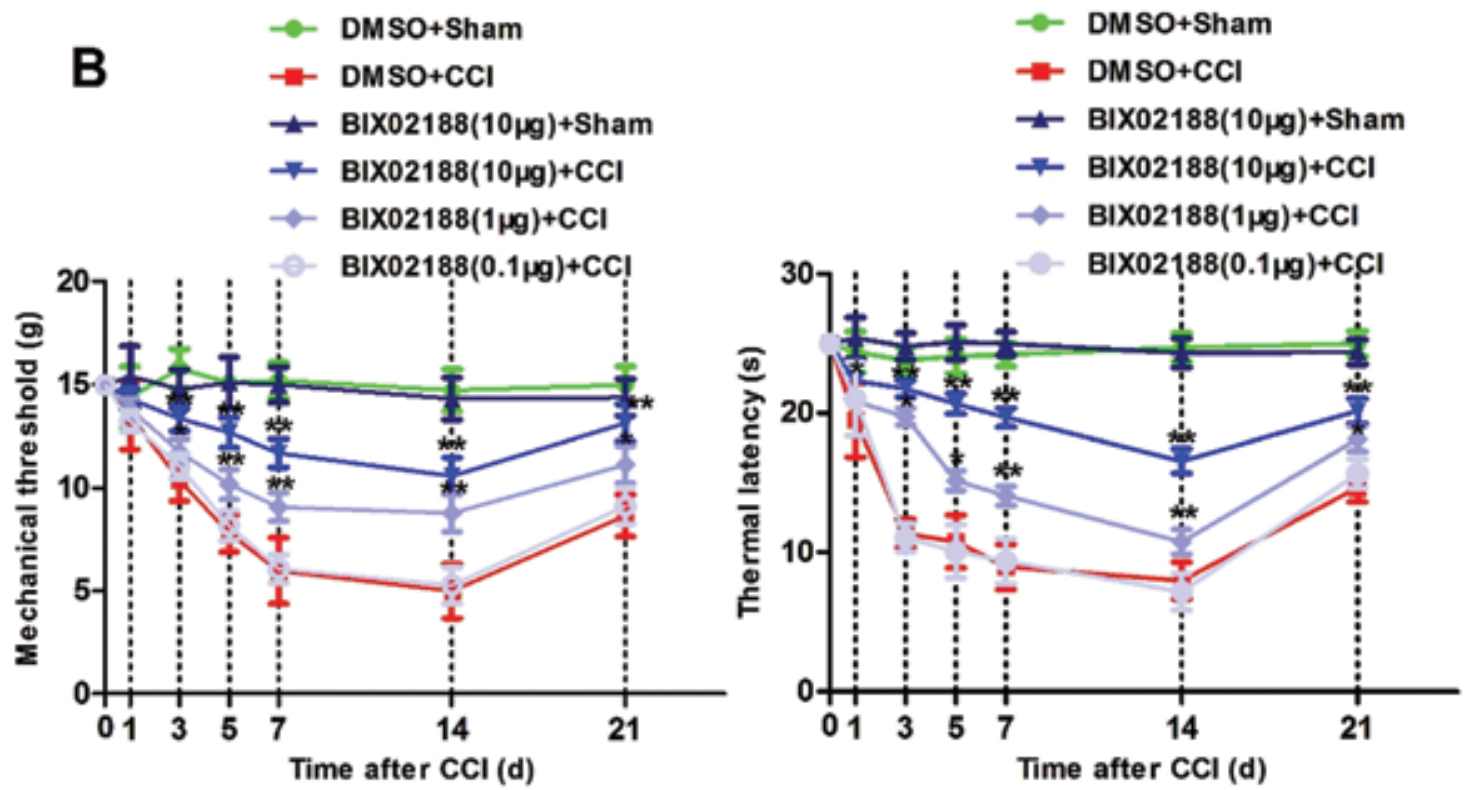

Fig. 3. Intra-lateral ventricles post- or pre-treatment with BIX02188 inhibited chronic constrictive injury-induced neuropathic pain. A: Post-treatment with BIX02188 (10 $\mu \mathrm{g})$ reversed CCI-induced mechanical allodynia in paw-withdrawal threshold $(P W T)$ test and thermal hyperalgesia in paw-withdrawal latency (PWL) test. One hour after intra- lateral ventricles infusion, the analgesic effect reached its peak, and then decreased gradually until hour $8 .{ }^{*} P<0.01,{ }^{*} P<0.05$ compared with DMSO+CCI group, $n=12$ rats in each group. B: Pre-treatment of BIX02188 $(0.1 \mu \mathrm{g}, 1 \mu \mathrm{g}, 10 \mu \mathrm{g})$ relieved CCI-induced mechanical allodynia and thermal hyperalgesia in a dose-dependent manner. ${ }^{*} * P<0.01,{ }^{*} P<0.05$ compared with DMSO+CCI group, $n=12$ rats in each group.

logical and certain special physiological conditions, the chemical composition of CSF can be changed, which was identified by clinical and animal experiments $(10,24-$ 25). Intercellular communication in the brain contains 2 types of transmission: wiring transmission by synaptic contacts and volume transmission, via the diffusion of messengers by the intercellular space or CSF (25-26). Our previous study indicated that there were excitatory and inhibitory synapses between the CSF-CN and nonCSF-CN by electronic microscope technology (10). The actual composition of CSF may be influenced by dCSFCNs for non-synaptic signal transmission via releasing or 

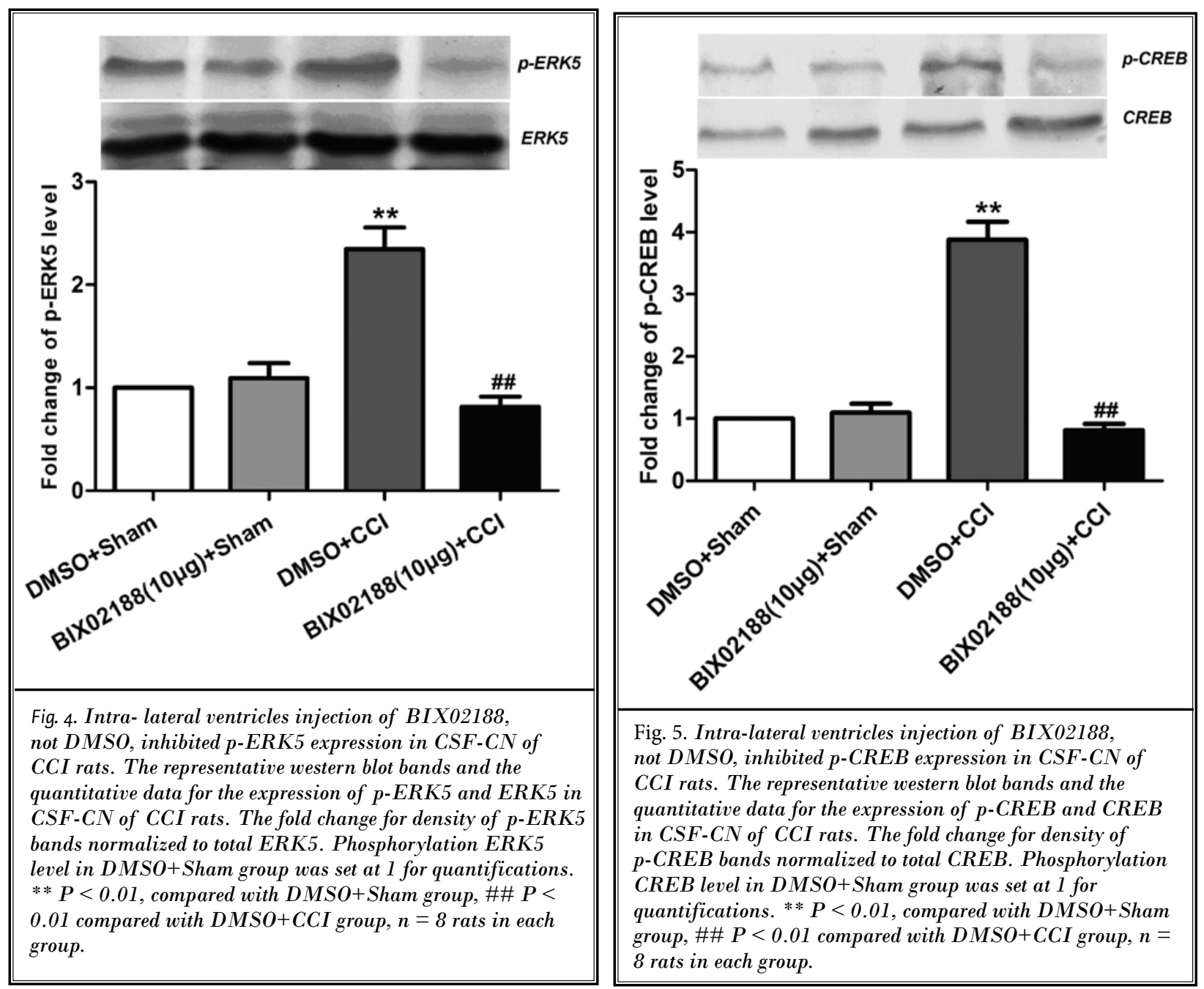

absorbing bioactive substances (26). However, CSF-CN can transmit the information to neurons of other areas in the brain via synaptic contacts. In this study, we found CB-HRP/p-ERK5 double-labeled neurons were increased significantly in CSF-CN of rats with nerve injury, which indicated that p-ERK5 in CSF-CN might regulate the information transfer in pathological conditions between brain parenchymal and CSF.

Neuropathic pain, considered as a multifactor neurogenic disorder, is a major public health issue, which can be caused by physical damage of neurons, cancer, and other diseases (27). Central sensitizations such as synaptic and neuronal plasticity are the neurobiological basis of behavior hyperalgesia under neuropathic conditions (28-30). Activation of intracellular kinase cascades, postsynaptic membrane receptors, or ion channels, and intranuclear gene expression contributes to the induc-

tion, development, and maintenance of central sensitization, especially mitogen-activated protein kinases (16). Compelling evidence indicates that MAPK play an important role in the development and maintenance of neuropathic pain. Activation of ERK is required for neuronal plasticity, such as learning and memory, and it also plays an important role in the transmission of pain sensation (31). Synapses, as the connection of dCSF-CNs, are the key structure of information transmission. Many peptides relevant to neuropathic pain, such as SP and calcitonin gene-related peptide (CGRP), are released from synaptic vesicles of dCSF-CNs, which may interact with their receptors leading to an augmentation of the discharge and long-term potentiation in dCSF-CNs. These peptides and their receptors activate ERK/MAPK by coupling to the ERK/MAPK (32-33). Activated ERK/ MAPK is translocated to nucleus and phosphorylate 
nuclear factor p90 ribosomal S6 kinase (RSK) which then activates CREB (34-35). Neuropathic pain-related genes, such as brain-derived neurotrophic factor (BDNF), calcitonin gene-related peptide (CGRP), the immediate early gene c-Fos, neurokinin 1 receptor, and the alpha subunit of calcium-calmodulin-dependent kinase (CaMK), might be activated by CAMP CREB and involved in synaptic plasticity underlying neuropathic pain (36-39). Our previous study showed p-ERK5 might contribute to inflammatory pain by regulating the activation of CREB (8). In this research we found ERK5 was activated in CSF-CN in nerve injury rats; besides these, intra-lateral ventricles injection of BIX02188 attenuated $\mathrm{CCl}$-induced mechanical allodynia and thermal hypersensitivity, which indicated that ERK5 in CSF-CN might be involved in neuropathic pain. However, considering selective inhibition of ERK5 activation only partially suppressed sensitization of pain, we presumed that neuropathic pain maybe a process involving a multisignaling pathway, and other neurotrophic factors or small protein might contribute to it. In addition, in this study, CCl-induced mechanical allodynia and thermal hypersensitivity significantly increased the activation of CREB in CSF-CN. However, intra-lateral ventricles injection of BIX02188 remarkably inhibited the expression of p-CREB in CSF-CN in CCl rats. Based on these findings, we proposed that activation of ERK5 might increase the expression of $p$-CREB in CSF-CN of neuropathic pain rats and the function of ERK5 might be partly regulated by CREB. These new findings extended our understanding about the role of ERK5 pathway in neuropathic pain.

\section{Conclusions}

In summary, we found that activation of ERK5 in CSF-CN might contribute to the development of neuropathic pain. Our findings pointed to the potential blockade of ERK5 signaling in CSF-CN as a new therapeutic strategy for neuropathic pain and its specific pharmacological inhibitor BIX02188 could have potential use for alleviation of mechanical allodynia and thermal hypersensitivity in the future.

\section{References}

1. Haanpaa M, Attal N, Backonja M, Baron R, Bennett $M$, Bouhassira D, Cruccu G, Hansson P, Haythornthwaite JA, lannetti GD, Jensen TS, Kauppila T,Nurmikko T), Rice AS, Rowbotham M, Serra J, Sommer C, Smith BH, Treede RD. NeuPSIG guidelines on neuropathic pain assessment. Pain 2011; 152:14-27.

2. Treede RD, Jensen TS, Campbell JN, Cruccu G, Dostrovsky JO, Griffin JW, Hansson $P$, Hughes R, Nurmikko T, Serra J. Neuropathic pain: Redefinition and a grading system for clinical and research purposes. Neurology 2008; 70:1630-1635

3. Lee JD, Ulevitch RJ, Han J. Primary structure of BMK1: A new mammalian map kinase. Biochem Biophys Res Commun 1995; 213:715-724.

4. Katsura H, Obata K, Mizushima T, , Kobayashi K, Yamanaka H, Dai Y, Fukuoka T, Sakagami M, Noguchi K. Activation of extracellular signal-regulated protein kinases 5 in primary afferent neurons contributes to heat and cold hyperalgesia after inflammation. J Neurochem 2007; 102:1614-1624.

5. Obata K, Katsura H, Mizushima T, Sakurai J, Kobayashi K, Yamanaka H, Dai Y, Fukuoka T, Noguchi K. Roles of extracellular signal-regulated protein ki- nases 5 in spinal microglia and primary sensory neurons for neuropathic pain. J Neurochem 2007; 102:1569-1584.

6. Wang CG, Ding YL, Zheng TF, Wei JQ, Liu H, Chen YF, Wang JY, Zhang LC. Extracellular signal-regulated kinase 5 in the cerebrospinal fluid-contacting nucleus contributes to morphine physical dependence in rats. J Mol Neurosci 2012; 50:215-220.

7. Wang CG, Lu XF, Wei JQ, Liu H, Zhang HX, Zhang LC, Cao JL. Activation of the spinal extracellular signal-regulated $\mathrm{ki}$ nase 5 signaling pathway contributes to morphine physical dependence in rats. Neurosci Lett 2011; 494:38-43.

8. Xiao C, Zhang L, Cheng QP, Zhang LC. The activation of extracellular signal-regulated protein kinase 5 in spinal cord and dorsal root ganglia contributes to inflammatory pain. Brain Res 2008; 1215:76-86.

9. Vigh-Teichmann I, Vigh B. The cerebrospinal fluid-contacting neuron: a peculiar cell type of the central nervous system. Immunocytochemical aspects. Arch Histol Cytol 1989; 52:195-207.

10. Zhang LC, Zeng YM, Ting J, Cao JP, Wang MS. The distributions and signaling directions of the cerebrospinal fluid con- tacting neurons in the parenchyma of a rat brain. Brain Res 2003; 989:1-8.

11. Lu XF, Li YY, Wang CG, Wei JQ, Ye $Y$, Zhang LC, Cao JL. Substance $P$ in the cerebrospinal fluid-contacting nucleus contributes to morphine physical dependence in rats. Neurosci Lett 2011; 488:188-192.

12. Gonzalez-Hernandez A, Martinez-Lorenzana G, Rojas-Piloni G, RodríguezJiménez J, Hernández-Linares Y, Villanueva L, Condés-Lara M. Spinal LTP induced by sciatic nerve electrical stimulation enhances posterior triangular thalamic nociceptive responses. Neuroscience 2013; 234C:125-134.

13. Gustin SM, Peck CC, Cheney LB, Macey PM, Murray GM, Henderson LA. Pain and plasticity: Is chronic pain always associated with somatosensory cortex activity and reorganization? J Neurosci 2012; 32:14874-14884.

14. King T, Qu C, Okun A, Melemedjian OK, Mandell EK, Maskaykina IY, Navratilova E, Dussor GO, Ghosh S, Price TJ, Porreca F. Contribution of PKMzeta-dependent and independent amplification to components of experimental neuropathic pain. Pain 2012; 153:1263-1273. 
15. Marcello L, Cavaliere C, Colangelo AM, Bianco MR, Cirillo G, Alberghina L, Papa M. Remodelling of supraspinal neuroglial network in neuropathic pain is featured by a reactive gliosis of the nociceptive amygdala. Eur J Pain 2012; 17:799-810.

16. Yu D, Thakor DK, Han I, Ropper AE, Haragopal H, Sidman RL, Zafonte $\mathrm{R}$, Schachter SC, Teng YD. Alleviation of chronic pain following rat spinal cord compression injury with multimodal actions of huperzine A. Proc Natl Acad Sci U S A 2013; 110:746-755.

17. Zhu XY, Huang CS, Li Q, Chang RM, Song ZB, Zou WY, Guo QL. P300 exerts an epigenetic role in chronic neuropathic pain through its acetyltransferase activity in rats following chronic constriction injury (CCl). Mol Pain 2012; 8:84.

18. Geng XJ, Lu XF, Zhang LC, Zeng YM. Expression of drebrin in the distal cerebrospinal fluid contacting neurons of rats with chronic constriction injury of sciatic nerve. Sheng Li Xue Bao 2008; 60:469-474

19. Lu X, Geng X, Zhang L, Zeng Y, Dong $H$, Yu $H$. Substance $P$ expression in the distal cerebrospinal fluid-contacting neurons and spinal trigeminal nucleus in formalin-induced the orofacial inflammatory pain in rats. Brain Res Bull 2009; 78:139-144

20. Chao CL, Lu XF, Zhang LC. Formalin-induced pain stimulation induced expression of GABA in the distal cerebrospinal fluid contacting neurons. Zhongguo Ying Yong Sheng Li Xue Za Zhi 2010; 26:36-38.

21. Zimmermann M. Ethical guidelines for investigations of experimental pain in conscious animals. Pain 1983; 16:109-110.

22. Bennett GJ, Xie YK. A peripheral mononeuropathy in rat that produces disorders of pain sensation like those seen in man. Pain 1988; 33:87-107.

23. Hargreaves K, Dubner R, Brown F, Flores $C$, Joris J. A new and sensitive method for measuring thermal nociception in cutaneous hyperalgesia. Pain 1988; 32:77-88.

24. Spaziante R, Merola B, Colao A, Gargiulo G, Cafiero T, Irace C, Rossi E, Oliver C, Lombardi G, Mazzarella B. Beta-endorphin concentrations both in plasma and in cerebrospinal fluid in response to acute painful stimuli. ] Neurosurg Sci 1990; 34:99-106.

25. Vigh B, Manzano e Silva MJ, Frank CL, Vincze C, Czirok SJ, Szabó A, Lukáts A, Szél A. The system of cerebrospinal fluid-contacting neurons. Its supposed role in the nonsynaptic signal transmission of the brain. Histol Histopathol 2004; 19:607-628.

26. Calle M, Claassen IE, Veening JG, Kozicz T, Roubos EW, Barendregt HP. Opioid peptides, CRF, and urocortin in cerebrospinal fluid-contacting neurons in Xenopus laevis. Ann N Y Acad Sci 2005; 1040:249-252.

27. Tou WI, Chang SS, Lee CC, Chen CY. Drug design for neuropathic pain regulation from traditional Chinese medicine. Sci Rep 2013; 3:844.

28. Ikeda H, Heinke B, Ruscheweyh R, Sandkühler J. Synaptic plasticity in spinal lamina I projection neurons that mediate hyperalgesia. Science 2003; 299:1237-1240.

29. Ji RR, Kohno T, Moore KA, Woolf CJ. Central sensitization and LTP: Do pain and memory share similar mechanisms? Trends Neurosci 2003; 26:696-705.

30. Melzack R, Coderre TJ, Katz J, Vaccarino AL. Central neuroplasticity and pathological pain. Ann N Y Acad Sci 2001; 933:157-174

31. Kiguchi N, Maeda T, Kobayashi Y, Fu- kazawa Y, Kishioka S. Activation of extracellular signal-regulated kinase in sciatic nerve contributes to neuropathic pain after partial sciatic nerve ligation in mice. Anesth Analg 2009; 109:1305-1311.

32. Cao JL, He JH, Ding HL, Zeng YM. Activation of the spinal ERK signaling pathway contributes naloxone-precipitated withdrawal in morphine-dependent rats. Pain 2005; 118:336-349.

33. Chen $Y$, Sommer $C$. The role of mitogen-activated protein kinase (MAPK) in morphine tolerance and dependence. Mol Neurobiol 2009; 40:101-107.

34. Xing J, Ginty DD, Greenberg ME. Coupling of the RAS-MAPK pathway to gene activation by RSK2, a growth factorregulated CREB kinase. Science 1996; 273:959-963.

35. Watson FL, Heerssen HM, Bhattacharyya A, Klesse L, Lin MZ, Segal RA. Neurotrophins use the Erk5 pathway to mediate a retrograde survival response. Nat Neurosci 2001; 4:981-988.

36. Wang Y, Cheng X, Xu J, Liu Z, Wan Y, Ma D. Anti-hyperalgesic effect of CaMKII inhibitor is associated with downregulation of phosphorylated CREB in rat spinal cord. J Anesth 2011; 25:87-92.

37. Trang T, Beggs S, Salter MW. Brainderived neurotrophic factor from microglia: A molecular substrate for neuropathic pain. Neuron Glia Biol 2011; 7:99-108.

38. Teodoro FC, Tronco Junior MF, Zampronio AR, Martini AC, Rae GA, Chichorro $J G$. Peripheral substance $P$ and neurokinin-1 receptors have a role in inflammatory and neuropathic orofacial pain models. Neuropeptides 2013; 47:199-206.

39. Bennett AD, Chastain KM, Hulsebosch CE. Alleviation of mechanical and thermal allodynia by $\operatorname{CGRP}(8-37)$ in a rodent model of chronic central pain. Pain 2000; 86:163-175. 
